\title{
A novel method for the synthesis of methylthiophenes from ketones containing an active methylene group
}

\author{
Michael M. Krayushkin,* Boris V. Lichitsky, Denis V. Kozhinov, Sergey N. Ivanov, and \\ Arkady A. Dudinov
}

N. D. Zelinsky Institute of Organic Chemistry, Russian Academy of Sciences, 47 Leninsky prosp., 119991 Moscow, Russian Federation

E-mail: mkray@ioc.ac.ru

\section{Dedicated to Professor B.A. Trofimov on the occasion of his $65^{\text {th }}$ birthday \\ (received 28 May 03; accepted 03 Sept 03; published on the web 03 Sept 03)}

\begin{abstract}
A method for the synthesis of methylthiophenes from various ketones has been elaborated. The target methylthiophenes were used for the synthesis of photochromic 1,2-dithienylethenes with heterocyclic bridges.
\end{abstract}

Keywords: Ketones, methylthiophenes, hydrides, reduction, photochromes

\section{Introduction}

Functionally substituted 1,2-dithienylethane derivatives are of great interest for the progress of the chemistry of photochromic 1,2-dihetero-arylethenes. ${ }^{1}$ 2-Methylthiophenes are widely used for the synthesis of photochromic dihetero-arylethenes as prospective elements of $3 \mathrm{D}$ optical memory. ${ }^{2-4}$ The most general and convenient method for the synthesis of methylthiophenes is the reduction of thiophene carboxaldehydes. ${ }^{5}$ The latter, however, are not always available, and for this reason the elaboration of novel methods for the synthesis of methylthiophenes is a problem.

\section{Results and Discussion}

We report a novel general three-step approach for the synthesis of the methylthiophenes $\mathbf{1}$ starting from active-methylene ketones. The first step in the scheme included heating the starting ketones 2 in $\mathrm{POCl}_{3} / \mathrm{DMF}$ at $40^{\circ} \mathrm{C}$ led to the chloro-aldehydes 3 in $50-99 \%$ yields. ${ }^{6}$ Reaction of the compounds 3 with methyl thioglycolate in methanolic $\mathrm{NaOMe}$ proceeded readily to provide the methyl thiophenecarboxylates 4 in $60-80 \%$ yields $^{7}$ (Scheme 1). We were interested in the 
transformation of the ester moiety into a methyl group. General methods of this kind are not present in the literature. Although there are a few examples of such a reduction, these methods are not sufficient. For example, pyrrole-3-carboxylic acid esters can be reduced to the corresponding 3-methylpyrroles by $\mathrm{LiAlH}_{4} .{ }^{8,9}$ Besides that, we have found three examples ${ }^{10-12}$ of one-pot synthesis of methyl derivatives from methyl carboxylates. The first example ${ }^{10}$ concerned the reduction of methyl anthranilate by $\mathrm{LiAlH}_{4}$ to methylaniline in $96 \%$ yield, but the process takes 20 days at room temperature. The second one ${ }^{11}$ deals with the reduction of cinnamic acid esters by a mixture of $\mathrm{LiAlH}_{4}$ and $\mathrm{AlCl}_{3}$. In this reaction, a mixture of the target compound and the product of allylic rearrangement was obtained in the ratio $2: 1$. The third example ${ }^{12}$ of reduction applied to fused heterocyclic system containing thiophene ring. However, the outlined procedures are not general, and cannot be applied to the reduction of other esters. Therefore it was necessary to elaborate a reliable procedure for the reduction of the obtained esters, 4 .

We have found that the esters 4 can be reduced by one-portion addition of $\mathrm{LiAlH}_{4}$ to their $\mathrm{AlCl}_{3} / \mathrm{Et}_{2} \mathrm{O}$ solutions. Reaction proceeded smoothly at room temperature and was complete in 2 hours. Work-up of the reaction mixture produced the methylthiophenes $\mathbf{1}$ in good yields. However, when dealing with the thiophenecarboxylates $\mathbf{4 f - g}$ we found that under the conditions we used, additional reduction of the bromine atom in the benzene ring occurred (Scheme 1).

Thus we have elaborated a three-step method for the synthesis of methylthiophenes starting from various ketones containing an active-methylene moiety. The method allows the synthesis of various methylthiophenes (fused, substituted) in good overall yields, under mild conditions. The thiophene 1b was used for the synthesis of 1,2-dithienylethenes with heterocyclic bridged fragments. Previously, we suggested methods for the synthesis of 1,2-dithienylethenes with heterocyclic bridges (e.g., furan ${ }^{13}$ and 1,3-dioxolan-2-one ${ }^{14}$ ) using 2,5-dimethylthiophene as starting material. It is $\mathrm{known}^{2}$ that the fatigue resistance of photochromic dithienylethenes is enhanced by the introduction of alkyl groups at position- 4 of the thiophene ring. We supposed that 1,2-dithienylethenes based on thiophene $\mathbf{1 b}$ would possess improved photochromic properties.

The 1,3-dioxolan-2-one fragment was selected as one of the most promising heterocyclic bridges. The method of synthesis of the dioxolan-2-one derivatives that we have elaborated ${ }^{14}$ was applied for the preparation of compound 5. Reaction of the acyloin 8 with excess of 1,1'carbonyldiimidazole leads to 4,5-di-(2-methyl-4,5,6,7-tetrahydrobenzo-[b]-thiophen-3-yl)-1,3dioxol-2-one 5 in high yield. (Scheme 2) The structure of compound 5 was confirmed using ${ }^{1} \mathrm{H}$ NMR spectroscopy, mass-spectrometry and elemental analysis data.

The photochromic characteristics of compound 5 were examined in acetonitrile solution. As was supposed, the dioxol-2-one 5 exhibits photochromic properties. The characteristic spectrum of the photochrome 5 is shown in Fig. 1. The long-wavelength absorption band of the open form A of compound 5 is observed at $229 \mathrm{~nm}$. The maximum of the first absorption band of the cyclic form of photochromic 1,3-dioxol-2-one 5 is observed at $453 \mathrm{~nm}$. The cyclic form of compound 5 is thermally irreversible, i.e., form $\mathbf{B}$ does not transform to form $\mathbf{A}$ without irradiation. It should 
be noted that this compound exhibits low fatigue resistance and the optical density of the cyclic form is regained by no more than $80 \%$ after three- to five-fold photochromic conversions.

To summarize, we have developed a procedure for the synthesis of substituted 2methylthiophenes which can be used for the preparation of photochromic dihetero-arylethenes. It was demonstrated that 1,3-dioxol-2-one 5 derived from 2-methyl-4,5,6,7-tetrahydrobenzo-[b]thiophene (1b) is the thermally irreversible photochromic compound.

(A)

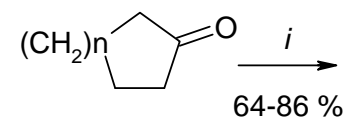<smiles>C[In]1CC(Cl)=C(C=O)C1</smiles>

2 a-c $n=1-3$

3 a-c
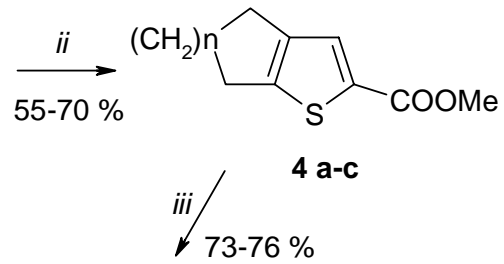

$\left(\mathrm{CH}_{2}\right)$<smiles>Cc1cc2c(s1)C[InH]C2</smiles>

1 a-c

(B)

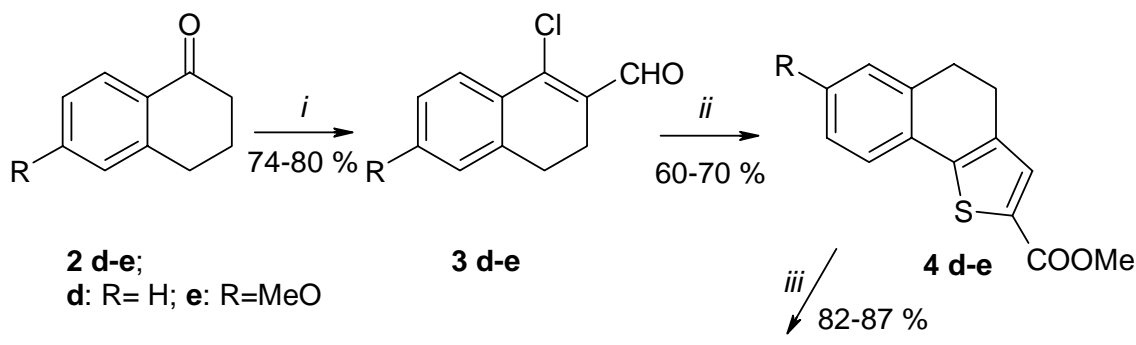<smiles>[R]c1ccc2c(c1)CCc1cc(C)sc1-2</smiles>

(C)

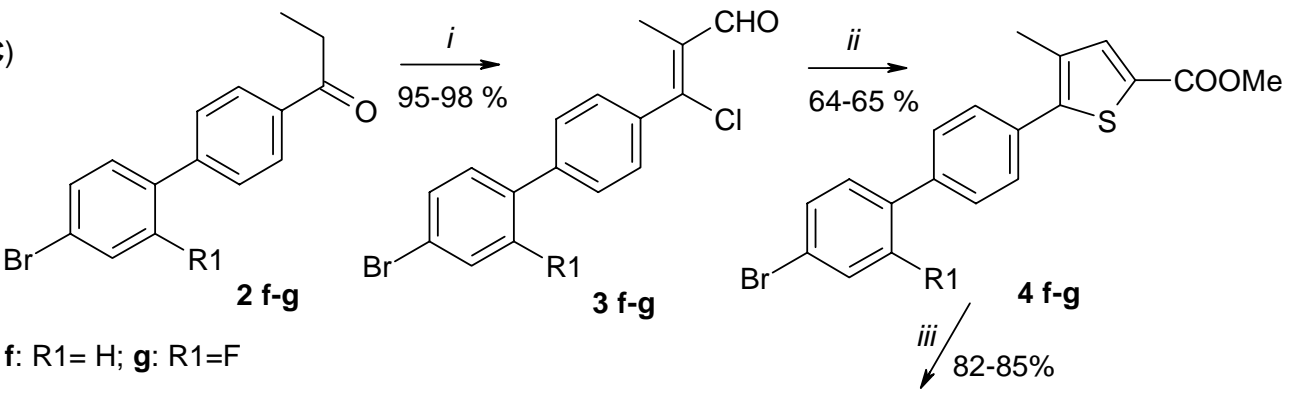

i: $\mathrm{POCl}_{3} / \mathrm{DMF} ;$ ii: $\mathrm{HSCH}_{2} \mathrm{COOMe}, \mathrm{NaOMe}, \mathrm{MeOH}$; iii: $\mathrm{LiAlH}_{4} / \mathrm{AlCl}_{3}, \mathrm{Et}_{2} \mathrm{O}$<smiles>[R1]c1ccccc1-c1ccc(-c2sc(C)cc2C)cc1</smiles>

\section{Scheme 1}




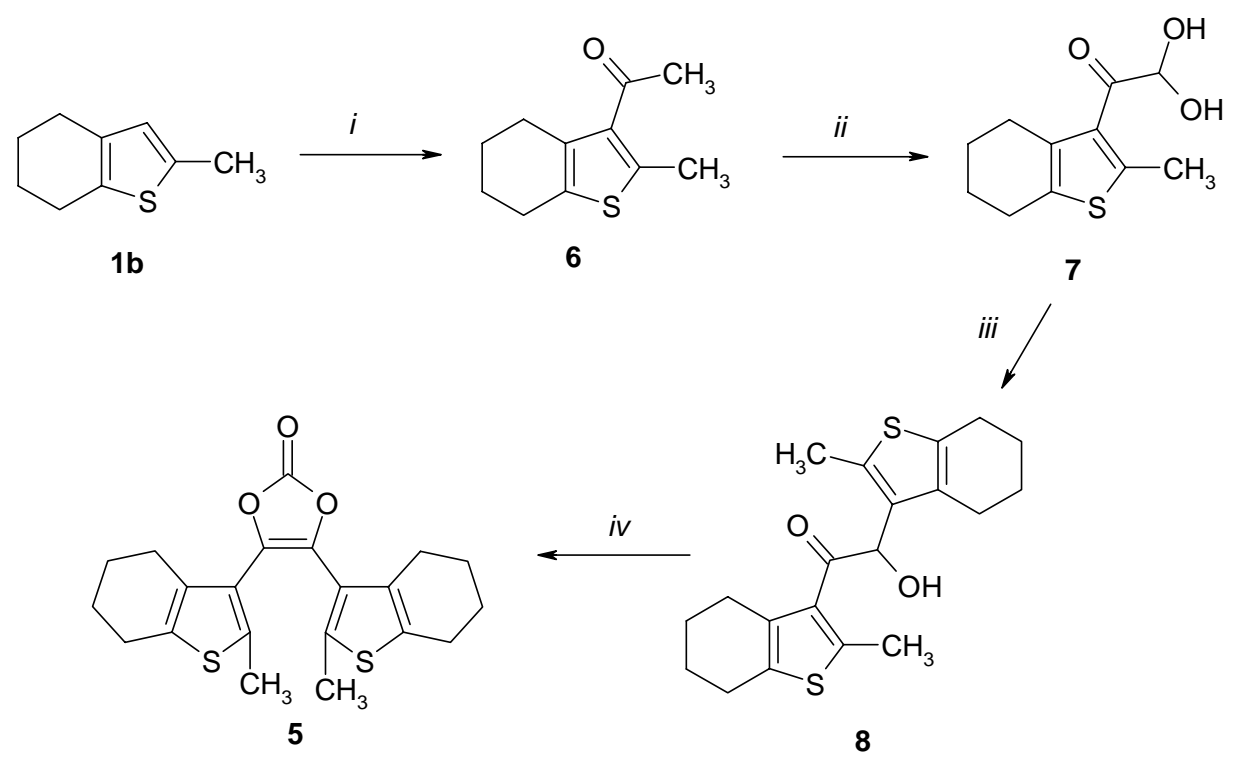

i: $\mathrm{AcCl}, \mathrm{SnCl}_{4}, \mathrm{CH}_{2} \mathrm{Cl}_{2}$; ii: $\mathrm{SeO}_{2}$, Dioxane ; iii: $\mathbf{1 b}, \mathrm{SnCl}_{4}, \mathrm{CH}_{2} \mathrm{Cl}_{2}$; iv: $\mathrm{CDI}, \mathrm{C}_{6} \mathrm{H}_{6}$

\section{Scheme 2}

\section{Experimental Section}

General Procedures. All reagents are commercially available (Aldrich, Acros) and were used without further purification. Column chromatography was performed on silica gel (Aldrich, grade 22, 60-200 mesh). Thin-layer chromatography (TLC, on aluminum plates coated with silica gel $60 \mathrm{~F}_{254}, 0.25 \mathrm{~mm}$ thickness, Merck) was used for monitoring the reactions; eluent hexane/ethyl acetate 6:1. Melting points (mp) were determined on a Kofler hot-stage microscope. ${ }^{1} \mathrm{H}$ NMR spectra were recorded with a Bruker AM-300 instrument (300.13 MHz). The mass spectrum was measured on a Kratos MS-30 instrument with direct inlet of the sample into the ion source; the energy of the ionizing electrons was $70 \mathrm{eV}$. Absorption spectra were obtained with a Shimadzu UV-3100 spectrophotometer in acetonitrile (special purity grade) solution at room temperature. Chloroaldehydes $\mathbf{4}$ were prepared as described. ${ }^{6,15}$ Ketone $\mathbf{6}$ was prepared from the corresponding thiophene $\mathbf{1 b}$ according to the reported procedure. ${ }^{3}$

The photochromic characteristics of compound 5 were studied in a solution in $\mathrm{MeCN}$ (special purity grade). The cyclic form $\mathbf{B}$ was prepared by irradiation of the sample with a DRSh500 mercury lamp using filters to separate lines of the $\operatorname{Hg}$ spectrum $(313,546$ and $578 \mathrm{~nm})$ and were then identified on the basis of $\lambda_{\max }$ in the UV spectrum. The intensity of radiation of the $\mathrm{Hg}$ lamp was determined using a F4 photoelement calibrated against a ferrioxalate actinometer for $\lambda$ $=313 \mathrm{~nm}$ and against an actinometer based on the Reinecke salt for $\lambda=546$ and $578 \mathrm{~nm}$. The absorption spectra were recorded on a Shimadzu UV-3100 spectrophotometer. 

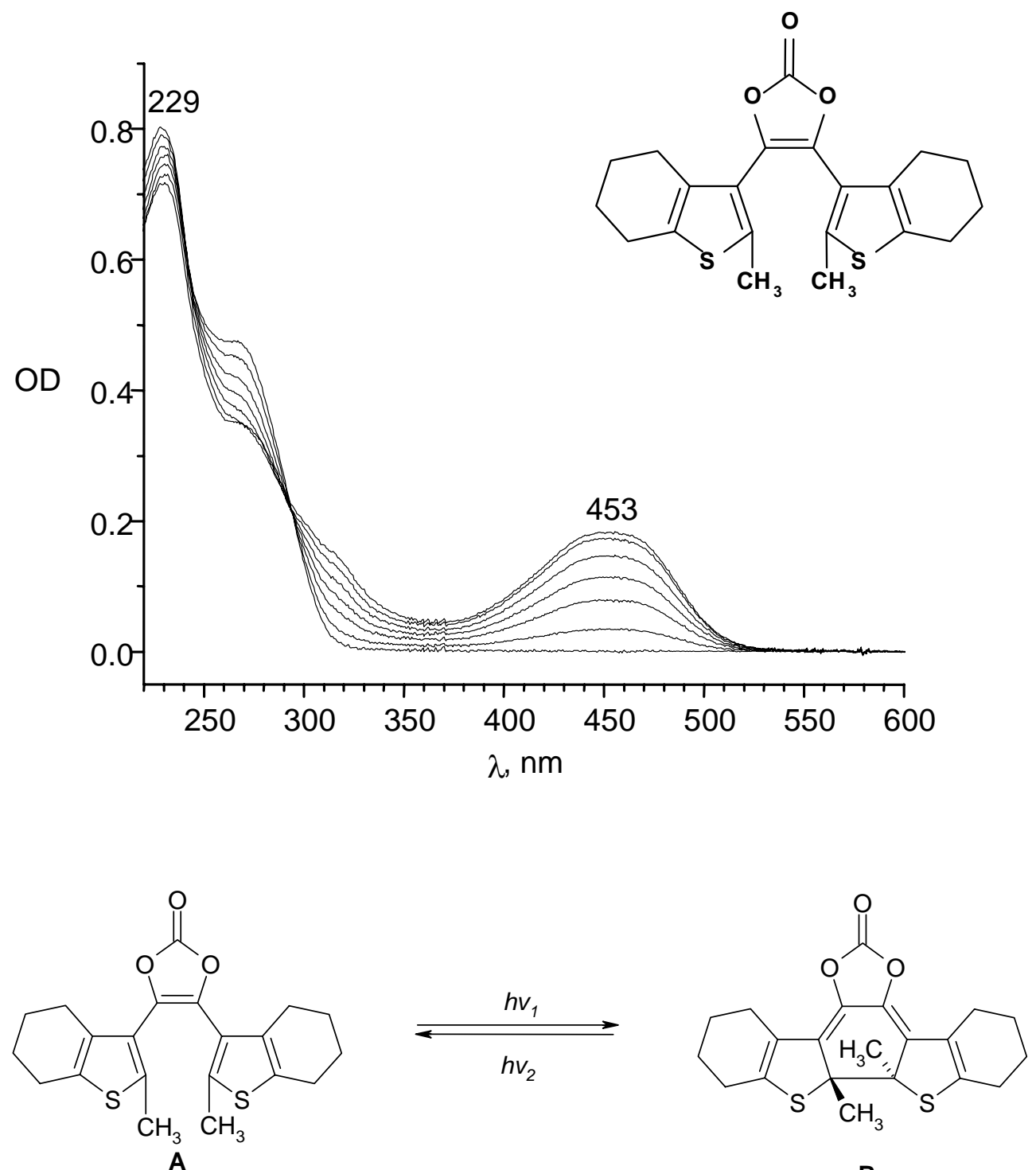

B

Figure 1. Changes in the absorption spectrum of 4,5-di-(2-methyl-4,5,6,7-tetrahydrobenzo-[b]thiophen-3-yl)-1,3-dioxol-2-one, 5, in a solution in acetonitrile before irradiation (open form A) and after irradiation (closed form B) with light at $\alpha 313 \mathrm{~nm}$.

\section{General method for the synthesis of ketones (2f-g)}

To a suspension of $\mathrm{AlCl}_{3}(31.5 \mathrm{mmol})$ in $\mathrm{CH}_{2} \mathrm{Cl}_{2}(70 \mathrm{ml})$ was added propionyl chloride (33 $\mathrm{mmol})$. The resulting mixture was stirred until the precipitate dissolved. Then the solution of the appropriate biphenyl $(30 \mathrm{mmol})$ in $\mathrm{CH}_{2} \mathrm{Cl}_{2}$ was added. The reaction mixture was stirred for $48 \mathrm{~h}$, and poured onto ice $(250 \mathrm{ml})$. The organic layer was separated, washed with water $(2 \times 50 \mathrm{ml})$, $\mathrm{NaHCO}_{3}(5 \%$ sol., $2 \times 50 \mathrm{ml})$ and dried over $\mathrm{MgSO}_{4}$. After evaporation of the solvent the residue was crystallized from EtOH $(40 \mathrm{ml})$. 
4-(4-Bromophenyl)propiophenone (2f). ${ }^{1} \mathrm{H}$ NMR (DMSO- $\left.\mathrm{d}_{6}\right): \delta 1.08$ (t, 3H, J $\left.7 \mathrm{~Hz}, \mathrm{CH}_{3}\right), 3.05$ (q, 2H, J $7 \mathrm{~Hz}, \mathrm{CH}_{2}$ ), 7.60-7.70 (m, 4H, $\mathrm{H}_{\mathrm{Ar}}$ ), 7.78 (d, 2H, J $8 \mathrm{~Hz}, \mathrm{H}_{\mathrm{Ar}}$ ), 8.02 (d, 2H, J $8 \mathrm{~Hz}, \mathrm{H}_{\mathrm{Ar}}$ ). Anal. Calcd. for $\mathrm{C}_{15} \mathrm{H}_{13} \mathrm{BrO}$ : C, 62.30; H, 4.53. Found: C, 62.37; H, 4.56\%.

4-(2-Fluoro-4-bromophenyl)propiophenone (2g). ${ }^{1} \mathrm{H}$ NMR (DMSO-d $\left.\mathrm{d}_{6}\right): \delta 1.10(\mathrm{t}, 3 \mathrm{H}, \mathrm{J} 7 \mathrm{~Hz}$, $\mathrm{CH}_{3}$ ), 3.02 (q, 2H, J $7 \mathrm{~Hz}, \mathrm{CH}_{2}$ ), 7.60-7.70 (m, 4H, $\mathrm{H}_{\mathrm{Ar}}$ ), 7.85-7.90 (m, 3H, $\mathrm{H}_{\mathrm{Ar}}$ ). Anal. Calcd. for $\mathrm{C}_{15} \mathrm{H}_{12} \mathrm{BrFO}$ : C, 58.65; H, 3.94. Found: C, 58.50; H, 3.87\%.

1-Chloro-6-methoxy-3,4-dihydro-2-naphthalenecarboxaldehyde (3e). ${ }^{1} \mathrm{H} \mathrm{NMR}\left(\mathrm{CDCl}_{3}\right): \delta$ $2.60\left(\mathrm{~m}, 2 \mathrm{H}, \mathrm{CH}_{2}\right), 2.80\left(\mathrm{~m}, 2 \mathrm{H}, \mathrm{CH}_{2}\right), 3.85\left(\mathrm{~s}, 3 \mathrm{H}, \mathrm{OCH}_{3}\right), 6.61\left(\mathrm{~s}, 1 \mathrm{H}, \mathrm{H}_{\mathrm{Ar}}\right), 6.82\left(\mathrm{~d}, 1 \mathrm{H}, \mathrm{H}_{\mathrm{Ar}}, \mathrm{J}\right.$ $8 \mathrm{~Hz}$ ), 7.78 (d, $\left.1 \mathrm{H}, \mathrm{H}_{\mathrm{Ar}}, \mathrm{J} 8 \mathrm{~Hz}\right), 10.30$ (s, 1H, CHO). Anal. Calcd. for $\mathrm{C}_{12} \mathrm{H}_{11} \mathrm{ClO}_{2}: \mathrm{C}, 64.73 ; \mathrm{H}$, 4.98. Found: C, 64.85; H, 4.95\%.

3-Chloro-2-methyl-3-[(4-bromophenyl)-4-phenyl]propenal (3f). ${ }^{1} \mathrm{H}$ NMR $\left(\mathrm{CDCl}_{3}\right): \delta 2.12$ (s, $\left.3 \mathrm{H}, \mathrm{CH}_{3}\right), 7.70-7.45\left(\mathrm{~m}, 8 \mathrm{H}, \mathrm{H}_{\mathrm{Ar}}\right), 9.56(\mathrm{~s}, 1 \mathrm{H}, \mathrm{CHO})$. Anal. Calcd. for $\mathrm{C}_{16} \mathrm{H}_{12} \mathrm{BrClO}$ : C, 57.26; H, 3.60. Found: C, 57.33; H, 3.56\%.

3-Chloro-2-methyl-3-[(2-fluoro-4-bromophenyl)-4-phenyl]propenal (3g). ${ }^{1} \mathrm{H}$ NMR $\left(\mathrm{CDCl}_{3}\right.$, $\delta): 9.58(\mathrm{~s}, 1 \mathrm{H}, \mathrm{CHO}), 7.60-7.30\left(\mathrm{~m}, 7 \mathrm{H}, \mathrm{H}_{\mathrm{Ar}}\right), 2.12\left(\mathrm{~s}, 3 \mathrm{H}, \mathrm{CH}_{3}\right)$. Anal. Calcd. For $\mathrm{C}_{16} \mathrm{H}_{11} \mathrm{BrClFO}$ : C, 54.35; H, 3.14. Found: C, 54.42; H, 3.11\%.

\section{General procedure for the synthesis of methylthiophene carboxylates (4)}

Sodium (1.2 g, $0.052 \mathrm{~mol})$ was dissolved in anhydrous $\mathrm{MeOH}(50 \mathrm{ml})$. Methyl thioglycolate $(2.7$ $\mathrm{ml}, 0.030 \mathrm{~mol})$ was added to this solution and stirred for $15 \mathrm{~min}$. Then aldehyde $3(0.026 \mathrm{~mol})$ was added over $30 \mathrm{~min}$. A water bath was used to maintain the reaction mixture at about $20-25{ }^{\circ} \mathrm{C}$. The mixture was then stirred overnight.

Work-up in the cases a-c: The reaction mixture was diluted with water $(200 \mathrm{ml})$ and extracted with ether $(3 \times 100 \mathrm{ml})$. The ether layer was washed with water $(3 \times 100)$, dried over $\mathrm{MgSO}_{4}$ and evaporated. The crude esters were distilled in vacuum $(\mathbf{4 b}-\mathbf{c})$ or recrystallized from hexane $(\mathbf{4 a})$. Work-up for cases $\mathbf{d}-\mathbf{g}$ : The reaction mixture was filtered and the residue washed with a large amount of water. The crude esters were recrystallized from $\mathrm{MeOH}(30 \mathrm{ml})$ to give the pure compounds 4.

Methyl 5,6-dihydro-4H-cyclopenta-[b]-thiophene-2-carboxylate (4a). ${ }^{1} \mathrm{H}$ NMR (DMSO- $\mathrm{d}_{6}$ ): $\delta 2.35\left(\mathrm{~m}, 2 \mathrm{H}, \mathrm{CH}_{2}\right), 2.70\left(\mathrm{~m}, 2 \mathrm{H}, \mathrm{CH}_{2}\right), 2.90\left(\mathrm{~m}, 2 \mathrm{H}, \mathrm{CH}_{2}\right), 3.79\left(\mathrm{~s}, 3 \mathrm{H}, \mathrm{OCH}_{3}\right), 7.52(\mathrm{~s}, 1 \mathrm{H}$, $\mathrm{H}_{\mathrm{Ar}}$ ). Anal. Calcd. For $\mathrm{C}_{9} \mathrm{H}_{10} \mathrm{O}_{2} \mathrm{~S}$ : C, 59.32; H, 5.53; S, 17.59. Found: C, 59.40; H, 5.50; S, $17.65 \%$.

Methyl 4,5,6,7-tetrahydrobenzo-[b]-thiophene-2-carboxylate (4b). ${ }^{1} \mathrm{H}$ NMR (DMSO-d 6 ): $\delta$ $1.70\left(\mathrm{~m}, 4 \mathrm{H}, 2 \mathrm{CH}_{2}\right), 2.55\left(\mathrm{~m}, 2 \mathrm{H}, \mathrm{CH}_{2}\right), 2.72\left(\mathrm{~m}, 2 \mathrm{H}, \mathrm{CH}_{2}\right), 3.75\left(\mathrm{~s}, 3 \mathrm{H}, \mathrm{OCH}_{3}\right), 7.45(\mathrm{~s}, 1 \mathrm{H}$, $\mathrm{H}_{\mathrm{Ar}}$ ). Anal. Calcd. For $\mathrm{C}_{10} \mathrm{H}_{12} \mathrm{O}_{2} \mathrm{~S}: \mathrm{C}, 61.20 ; \mathrm{H}, 6.16$; S, 16.34. Found: C, 61.35; H, 6.20; $\mathrm{S}$, $16.26 \%$.

Methyl 5,6,7,8-tetrahydro-4H-cyclohepta-[b]-thiophene-2-carboxylate (4c). ${ }^{1} \mathrm{H} \quad \mathrm{NMR}$ (DMSO-d $\left.)_{6}\right): \delta 1.60\left(\mathrm{~m}, 4 \mathrm{H}, 2 \mathrm{CH}_{2}\right), 1.80\left(\mathrm{~m}, 2 \mathrm{H}, \mathrm{CH}_{2}\right), 2.70\left(\mathrm{~m}, 2 \mathrm{H}, \mathrm{CH}_{2}\right), 2.82\left(\mathrm{~m}, 2 \mathrm{H}, \mathrm{CH}_{2}\right)$, $3.75\left(\mathrm{~s}, 3 \mathrm{H}, \mathrm{OCH}_{3}\right), 7.51\left(\mathrm{~s}, 1 \mathrm{H}, \mathrm{H}_{\mathrm{Ar}}\right)$. Anal. Calcd. For $\mathrm{C}_{11} \mathrm{H}_{14} \mathrm{O}_{2} \mathrm{~S}: \mathrm{C}, 62.83 ; \mathrm{H}, 6.71 ; \mathrm{S}, 15.25$. Found: C, 62.98; H, 6.67; S, 15.14\%. 
Methyl 4,5-dihydronaphtho-[1,2-b]-thiophene-2-carboxylate (4d). ${ }^{1} \mathrm{H}$ NMR (DMSO-d 6 ): $\delta$ $2.80\left(\mathrm{~m}, 2 \mathrm{H}, \mathrm{CH}_{2}\right), 2.90\left(\mathrm{~m}, 2 \mathrm{H}, \mathrm{CH}_{2}\right), 3.80\left(\mathrm{~s}, 3 \mathrm{H}, \mathrm{OCH}_{3}\right), 7.35\left(\mathrm{~m}, 3 \mathrm{H}, \mathrm{H}_{\mathrm{Ar}}\right), 7.42\left(\mathrm{~m}, 1 \mathrm{H}, \mathrm{H}_{\mathrm{Ar}}\right)$, 7.66 (s, $1 \mathrm{H}, \mathrm{H}_{\mathrm{Ar}}$ ). Anal. Calcd. For $\mathrm{C}_{14} \mathrm{H}_{12} \mathrm{O}_{2} \mathrm{~S}$ : C, 68.83; H, 4.95; S, 13.12. Found: C, 68.77; H, 4.99; S, 13.21\%.

Methyl 7-methoxy-4,5-dihydronaphtho-[1,2-b]-thiophene-2-carboxylate (4e). ${ }^{1} \mathrm{H} \quad \mathrm{NMR}$ $\left(\mathrm{DMSO}_{-} \mathrm{d}_{6}\right): \delta 2.75\left(\mathrm{~m}, 2 \mathrm{H}, \mathrm{CH}_{2}\right), 2.90\left(\mathrm{~m}, 2 \mathrm{H}, \mathrm{CH}_{2}\right), 3.77\left(\mathrm{~s}, 3 \mathrm{H}, \mathrm{OCH}_{3}\right), 3.82\left(\mathrm{~s}, 3 \mathrm{H}, \mathrm{OCH}_{3}\right)$, $6.80\left(\mathrm{~d}, 1 \mathrm{H}, \mathrm{H}_{\mathrm{Ar}}, \mathrm{J} 8 \mathrm{~Hz}\right), 6.91\left(\mathrm{~s}, 1 \mathrm{H}, \mathrm{H}_{\mathrm{Ar}}\right), 7.45\left(\mathrm{~d}, 1 \mathrm{H}, \mathrm{H}_{\mathrm{Ar}}, \mathrm{J} 8 \mathrm{~Hz}\right), 7.64\left(\mathrm{~s}, 1 \mathrm{H}, \mathrm{H}_{\mathrm{Ar}}\right)$. Anal. Calcd. For $\mathrm{C}_{15} \mathrm{H}_{14} \mathrm{O}_{3} \mathrm{~S}$ : C, 65.67; H, 5.14; S, 11.69. Found: C, 65.82; H, 5.10; S, $11.78^{\circ}$.

Methyl 4-methyl-5-[4-(4-bromophenyl)phenyl]-thiophene-2-carboxylate (4f). ${ }^{1} \mathrm{H} \quad \mathrm{NMR}$ $\left(\mathrm{DMSO}_{\mathrm{d}}\right): \delta 2.3\left(\mathrm{~s}, 3 \mathrm{H}, \mathrm{CH}_{3}\right), 3.80\left(\mathrm{~s}, 3 \mathrm{H}, \mathrm{OCH}_{3}\right), 7.70-7.55\left(\mathrm{~m}, 9 \mathrm{H}, \mathrm{H}_{\mathrm{Ar}}\right)$. Anal. Calcd. For $\mathrm{C}_{19} \mathrm{H}_{16} \mathrm{O}_{2} \mathrm{~S}$ : C, 74.00; H, 5.23; Br, 20.63; S, 8.28. Found: C, 73.91; H, 5.22; Br, 20.71; S, 8.20\%.

Methyl 4-methyl-5-[4-(2-fluoro-4-bromophenyl)phenyl]-thiophene-2-carboxylate (4g). ${ }^{1} \mathrm{H}$ NMR (DMSO-d $\left.{ }_{6}\right): \delta 2.32\left(\mathrm{~s}, 3 \mathrm{H}, \mathrm{CH}_{3}\right), 3.80\left(\mathrm{~s}, 3 \mathrm{H}, \mathrm{OCH}_{3}\right), 7.72-7.50\left(\mathrm{~m}, 8 \mathrm{H}, \mathrm{H}_{\mathrm{Ar}}\right)$. Anal. Calcd. For $\mathrm{C}_{19} \mathrm{H}_{15} \mathrm{FO}_{2} \mathrm{~S}$ : C, 69.92; H, 4.63. Found: C, 70.05; H, 4.69\%.

\section{General procedure for the synthesis of thiophenes (1a-e)}

To a solution of $\mathrm{AlCl}_{3}(3.0 \mathrm{~g}, 0.022 \mathrm{~mol})$ in anhydrous $\mathrm{Et}_{2} \mathrm{O}(100 \mathrm{ml})$ was added the corresponding ester $4(0.005 \mathrm{~mol})$. $\mathrm{LiAlH}_{4}(1.5 \mathrm{~g}, 0.038 \mathrm{~mol})$ was added to the solution in one portion. This was stirred for 2 hours, benzene $(100 \mathrm{ml})$ was added and the reaction mixture was carefully quenched with water $(50 \mathrm{ml})$. The organic layer was separated, washed with water ( $3 \times 100 \mathrm{ml})$, dried over $\mathrm{MgSO}_{4}$ and evaporated. The resulting thiophenes 1 were either distilled in vacuum $(\mathbf{1 a}-\mathbf{c})$ or purified on a column $(\mathbf{1 d}, \mathbf{e})$.

2-Methyl-5,6-dihydro-4H-cyclopenta-[b]-thiophene (1a). ${ }^{1} \mathrm{H} \mathrm{NMR}\left(\mathrm{CDCl}_{3}\right): \delta 2.40(\mathrm{~m}, 2 \mathrm{H}$, $\left.\mathrm{CH}_{2}\right), 2.47\left(\mathrm{~s}, 3 \mathrm{H}, \mathrm{CH}_{3}\right), 2.70\left(\mathrm{~m}, 2 \mathrm{H}, \mathrm{CH}_{2}\right), 2.88\left(\mathrm{~m}, 2 \mathrm{H}, \mathrm{CH}_{2}\right), 6.51\left(\mathrm{~s}, 1 \mathrm{H}, \mathrm{H}_{\mathrm{Ar}}\right)$.

2-Methyl-4,5,6,7-tetrahydrobenzo-[b]-thiophene (1b). ${ }^{1} \mathrm{H}$ NMR (DMSO- $\left.\mathrm{d}_{6}\right): \delta 1.70(\mathrm{~m}, 4 \mathrm{H}$, $\left.2 \mathrm{CH}_{2}\right), 2.30\left(\mathrm{~s}, 3 \mathrm{H}, \mathrm{CH}_{3}\right), 2.45\left(\mathrm{~m}, 2 \mathrm{H}, \mathrm{CH}_{2}\right), 2.60\left(\mathrm{~m}, 2 \mathrm{H}, \mathrm{CH}_{2}\right), 6.40\left(\mathrm{~s}, 1 \mathrm{H}, \mathrm{H}_{\mathrm{Ar}}\right)$.

2-Methyl 5,6,7,8-tetrahydro-4H-cyclohepta-[b]-thiophene (1c). ${ }^{1} \mathrm{H} \mathrm{NMR}\left(\mathrm{CDCl}_{3}\right): \delta 1.70(\mathrm{~m}$, $\left.4 \mathrm{H}, 2 \mathrm{CH}_{2}\right), 1.85\left(\mathrm{~m}, 2 \mathrm{H}, \mathrm{CH}_{2}\right), 2.38\left(\mathrm{~s}, 3 \mathrm{H}, \mathrm{CH}_{3}\right), 2.62\left(\mathrm{~m}, 2 \mathrm{H}, \mathrm{CH}_{2}\right), 2.75\left(\mathrm{~m}, 2 \mathrm{H}, \mathrm{CH}_{2}\right), 6.42(\mathrm{~s}$, $\left.1 \mathrm{H}, \mathrm{H}_{\mathrm{Ar}}\right)$.

2-Methyl 4,5-dihydronaphtho-[1,2-b]-thiophene (1d). ${ }^{1} \mathrm{H} \mathrm{NMR}\left(\mathrm{CDCl}_{3}\right): \delta 2.55\left(\mathrm{~s}, 3 \mathrm{H}, \mathrm{CH}_{3}\right)$, $2.80\left(\mathrm{~m}, 2 \mathrm{H}, \mathrm{CH}_{2}\right), 2.95\left(\mathrm{~m}, 2 \mathrm{H}, \mathrm{CH}_{2}\right), 6.60$ (s, 1H, $\left.\mathrm{H}_{\mathrm{Ar}}\right), 7.80-7.60$ (m, 4H, $\left.\mathrm{H}_{\mathrm{Ar}}\right)$. Anal. Calcd. For $\mathrm{C}_{13} \mathrm{H}_{12} \mathrm{~S}$ : C, 77.95; H, 6.04; S, 16.01. Found: C, 78.07; H, 6.01; S, 15.92\%.

2-Methyl-7-methoxy-4,5-dihydronaphtho-[1,2-b]-thiophene (1e). ${ }^{1} \mathrm{H}$ NMR $\left(\mathrm{CDCl}_{3}\right): \delta 2.50$ (s, 3H, $\left.\mathrm{CH}_{3}\right), 2.75\left(\mathrm{~m}, 2 \mathrm{H}, \mathrm{CH}_{2}\right), 2.92\left(\mathrm{~m}, 2 \mathrm{H}, \mathrm{CH}_{2}\right), 3.82\left(\mathrm{~s}, 3 \mathrm{H}, \mathrm{OCH}_{3}\right), 6.56\left(\mathrm{~s}, 1 \mathrm{H}, \mathrm{H}_{\mathrm{Ar}}\right), 6.75$ $\left(\mathrm{d}, 1 \mathrm{H}, \mathrm{H}_{\mathrm{Ar}}, \mathrm{J} 8 \mathrm{~Hz}\right), 6.78\left(\mathrm{~s}, 1 \mathrm{H}, \mathrm{H}_{\mathrm{Ar}}\right), 7.25$ (d, 1H, $\left.\mathrm{H}_{\mathrm{Ar}}, \mathrm{J} 8 \mathrm{~Hz}\right)$. Anal. Calcd. For $\mathrm{C}_{14} \mathrm{H}_{14} \mathrm{OS}$ : C, 73.01; H, 6.13; S, 13.92. Found: C, 73.10; H, 6.17; S, 13.79\%.

\section{General procedure for the synthesis of thiophenes $(1 \mathrm{f}-\mathrm{g})$}

To a solution of $\mathrm{AlCl}_{3}(2.0 \mathrm{~g}, 0.015 \mathrm{~mol})$ in anhydrous $\mathrm{Et}_{2} \mathrm{O}(70 \mathrm{ml})$ was added the corresponding ester $4(0.5 \mathrm{mmol})$. After dissolution of the latter, $\mathrm{LiAlH}_{4}(1.0 \mathrm{~g}, 0.025 \mathrm{~mol})$ was 
added in one portion. This was stirred for $2 \mathrm{~h}$, benzene $(100 \mathrm{ml})$ added, and the reaction mixture carefully quenched with water $(50 \mathrm{ml})$. The organic layer was separated, washed with water $(3 \times 100 \mathrm{ml})$, dried over $\mathrm{MgSO}_{4}$, and evaporated. The resulting thiophenes $\mathbf{1} \mathbf{f}-\mathbf{g}$ were recrystallized from methanol $(10 \mathrm{ml})$.

2,4-Dimethyl-5-biphenylthiophene (1f). ${ }^{1} \mathrm{H}$ NMR (DMSO- $\left.\mathrm{d}_{6}\right): \delta 2.25\left(\mathrm{~s}, 3 \mathrm{H}, \mathrm{CH}_{3}\right), 2.43(\mathrm{~s}, 3 \mathrm{H}$, $\left.\mathrm{CH}_{3}\right), 6.70(\mathrm{~s}, \mathrm{H}, \mathrm{CH}), 7.35-7.52\left(\mathrm{~m}, 5 \mathrm{H}, \mathrm{H}_{\mathrm{Ar}}\right), 7.65-7.75\left(\mathrm{~m}, 4 \mathrm{H}, \mathrm{H}_{\mathrm{Ar}}\right)$.

Anal. Calcd. For $\mathrm{C}_{18} \mathrm{H}_{16} \mathrm{~S}$ : C, 81.77; H, 6.10; S, 10.40. Found: C, 81.89; H, 6.02; S, 10.50\%.

2,4-Dimethyl-5-[4-(2-fluorophenyl)phenyl]thiophene (1g). ${ }^{1} \mathrm{H}$ NMR $\left(\mathrm{DMSO}-\mathrm{d}_{6}\right): \delta 2.32$ (s, $\left.3 \mathrm{H}, \mathrm{CH}_{3}\right), 2.50\left(\mathrm{~s}, 3 \mathrm{H}, \mathrm{CH}_{3}\right), 6.62\left(\mathrm{~s}, 1 \mathrm{H}, \mathrm{H}_{\mathrm{Ar}}\right), 7.70-7.10\left(\mathrm{~m}, 8 \mathrm{H}, \mathrm{H}_{\mathrm{Ar}}\right)$.

Anal. Calcd. For $\mathrm{C}_{18} \mathrm{H}_{15} \mathrm{FS}$ : C, 76.56; H, 5.35. Found: C, 76.45; H, 5.31\%.

2-(2-Methyl-4,5,6,7-tetrahydrobenzo-[b]-thiophen-3-yl)-2-oxoacetaldehyde (7). To a solution of selenium dioxide $(1.90 \mathrm{~g}, 0.017 \mathrm{~mol})$ in dioxane $(50 \mathrm{ml})$ and $\mathrm{H}_{2} \mathrm{O}(2 \mathrm{ml})$ at $60{ }^{\circ} \mathrm{C}$ was added ketone $6^{5}$ ( $\left.2.50 \mathrm{~g}, 0.013 \mathrm{~mol}\right)$. The mixture was refluxed for $8 \mathrm{~h}$, filtered (removal of Se), and the filtrate evaporated under reduced pressure. The oily residue thus obtained was crystallized from $\mathrm{H}_{2} \mathrm{O}$. The precipitate was removed by filtration, and washed with a small amount of water, affording colorless crystals of the hydrate of the ketoaldehyde $7(2.00 \mathrm{~g}, 68 \%), \mathrm{mp} 109-110{ }^{\circ} \mathrm{C}$. ${ }^{1} \mathrm{H}$ NMR (300 MHz, DMSO-d 6 ): $\delta$ 1.58-1.81 (5 H, m, $\left.5 \mathrm{CH}\right) ; 2.55-2.63(3 \mathrm{H}, \mathrm{m}, 3 \mathrm{CH}) ; 2.73$ (3 $\left.\mathrm{H}, \mathrm{s}, \mathrm{CH}_{3}\right) ; 5.42(1 \mathrm{H}, \mathrm{t}, J 8.0 \mathrm{~Hz}, \mathrm{CH}) ; 6.48(2 \mathrm{H}, \mathrm{d}, J 8.0 \mathrm{~Hz}, 2 \mathrm{OH})$. Anal. Calcd. For $\mathrm{C}_{11} \mathrm{H}_{12} \mathrm{O}_{2} \mathrm{~S} \cdot \mathrm{H}_{2} \mathrm{O}$ : C, 58.38; H, 6.24; S, 14.17. Found: C, 58.45; H, 6.31; S, 14.29\%.

2-Hydroxy-1,2-di-(2-methyl-4,5,6,7-tetrahydrobenzo-[b]-thiophen-3-yl)-1-ethanone (8). A solution of tin tetrachloride $(0.78 \mathrm{~g}, 0.003 \mathrm{~mol})$ in benzene $(5 \mathrm{ml})$ was added dropwise to a stirred solution of hydrate $7(0.45 \mathrm{~g}, 0.002 \mathrm{~mol})$ and thiophene $\mathbf{1 b}(0.35 \mathrm{~g}, 0.0023 \mathrm{~mol})$ in benzene $(10 \mathrm{ml})$. The reaction mixture was stirred for $3 \mathrm{~h}$ at room temperature. Then the solution was carefully poured into water $(50 \mathrm{ml})$ and extracted with ether $(3 \times 20 \mathrm{ml})$. The organic layer was separated, washed with water $(3 \times 50 \mathrm{ml})$, dried over $\mathrm{MgSO}_{4}$, and evaporated. The crude product was recrystallized from EtOH to give colorless crystals of acyloin, 8, $(0.60 \mathrm{~g}, 83 \%), \mathrm{mp}$ 146-147 ${ }^{\circ} \mathrm{C} .{ }^{1} \mathrm{H}$ NMR $\left(300 \mathrm{MHz}, \mathrm{CDCl}_{3}\right): \delta 1.51-1.85(10 \mathrm{H}, \mathrm{m}, 10 \mathrm{CH}) ; 2.15\left(3 \mathrm{H}, \mathrm{s}, \mathrm{CH}_{3}\right)$; $2.26\left(3 \mathrm{H}, \mathrm{s}, \mathrm{CH}_{3}\right) ; 2.44-2.85(6 \mathrm{H}, \mathrm{m}, 6 \mathrm{CH}) ; 4.31(1 \mathrm{H}, \mathrm{s}, 1 \mathrm{CH}) ; 5.54(1 \mathrm{H}, \mathrm{s}, 1 \mathrm{OH})$. Anal. Calcd. For $\mathrm{C}_{20} \mathrm{H}_{24} \mathrm{O}_{2} \mathrm{~S}_{2}$ : C, 66.63; H, 6.71; S, 17.79. Found: C, 66.56; H, 6.79; S, 17.92\%.

4,5-Di-(2-methyl-4,5,6,7-tetrahydrobenzo-[b]-thiophen-3-yl)-1,3-dioxol-2-one (5). A mixture of thenoin 8 ( $0.36 \mathrm{~g}, 0.001 \mathrm{~mol})$ and 1,1'-carbonyldiimidazole $(0.24 \mathrm{~g}, 0.015 \mathrm{~mol})$ in benzene (5 $\mathrm{ml}$ ) was heated at reflux for $10 \mathrm{~h}$. The reaction mixture was cooled and washed with water, $10 \%$ $\mathrm{HCl}$ solution, then water. The solvent was evaporated under reduced pressure and the oily residue thus obtained was crystallized from EtOH. The crystalline product was filtered off, and washed on the filter funnel with a small amount of EtOH, affording colorless crystals of product $5(0.34 \mathrm{~g}, 87 \%), \mathrm{mp} 117-118{ }^{\circ} \mathrm{C} .{ }^{1} \mathrm{H} \mathrm{NMR}\left(300 \mathrm{MHz}, \mathrm{CDCl}_{3}\right): \delta 1.62-1.87(8 \mathrm{H}, \mathrm{m}, 8 \mathrm{CH})$; $2.00\left(6 \mathrm{H}, \mathrm{s}, 2 \mathrm{CH}_{3}\right) ; 2.39(4 \mathrm{H}, \mathrm{m}, 4 \mathrm{CH}) ; 2.68(4 \mathrm{H}, \mathrm{m}, 4 \mathrm{CH}) . \mathrm{MS}(\mathrm{EI}), \mathrm{m} / \mathrm{z}\left[\mathrm{I}_{\text {rel }}(\%)\right]: 386\left(\mathrm{M}^{+}\right)$ (91). Anal. Calcd. For $\mathrm{C}_{21} \mathrm{H}_{22} \mathrm{O}_{3} \mathrm{~S}_{2}$ : C, 65.26; H, 5.74; S, 16.59. Found: C, 65.39; H, 5.68; S, $16.77 \%$. 
Table 1. Yields and physical properties of compounds synthesized

\begin{tabular}{cccc}
\hline Structure & Yield, $\%$ & Melting or boiling point, ${ }^{\circ} \mathrm{C}$ & Lit. ref. \\
\hline 1a & 73 & $94-95 / 20$ mbar & 16 \\
1b & 74 & $96-97 / 12 \mathrm{mbar}$ & 5 \\
1c & 76 & $124-125 / 18 \mathrm{mbar}$ & 17 \\
$\mathbf{1 d}$ & 82 & Oil & \\
$\mathbf{1 e}$ & 87 & Oil & \\
$\mathbf{1 f}$ & 85 & $86-87$ & \\
$\mathbf{1 g}$ & 82 & $87-88$ & \\
$\mathbf{2 f}$ & 85 & $121-122$ & \\
$\mathbf{2 g}$ & 73 & $104-105$ & \\
$\mathbf{3 e}$ & 74 & $76-77$ & \\
$\mathbf{3 f}$ & 98 & $137-138$ & \\
$\mathbf{3 g}$ & 95 & $104-105$ & \\
$\mathbf{4 a}$ & 55 & $59-60$ & \\
$\mathbf{4 b}$ & 70 & $149-150 / 10 \mathrm{mbar}$ & \\
$\mathbf{4 c}$ & 67 & b.p. 180-181/18 mbar; m.p. 4344 & \\
$\mathbf{4 d}$ & 60 & $82-83$ & \\
$\mathbf{4 e}$ & 70 & $83-84$ & \\
$\mathbf{4 f}$ & 64 & $132-134$ & \\
$\mathbf{4 g}$ & 65 & $133-135$ & \\
$\mathbf{5}$ & 87 & $117-118$ & \\
$\mathbf{7}$ & 68 & $109-110$ & \\
$\mathbf{8}$ & 83 & $146-147$ & \\
\hline
\end{tabular}

\section{References}

1. Krayushkin, M. M. Chem. Heterocyclic Comp. 2001, 1, 19.

2. Irie, M. Chem. Rev. 2000, 100, 1685.

3. Takami, S.; Kawai, T.; Irie, M. Eur. J. Org. Chem. 2002, 22, 3796.

4. Myles, A. J.; Branda, N. R. Advanced Functional Materials 2002, 12, 167.

5. Buu-Hoi, N. P.; Khenissi, M. Bull. Soc. Chim. Fr. 1958, 359.

6. Arnold, Z.; Zemlicka, J. Coll. Czech. Chem. Com. 1959, 2385.

7. Beaton, C. M.; Chapman, N. B.; Clarke, K.; Willis, J.-M. J. Chem. Soc., Perkin Trans. I 1976, 2355.

8. Treibs, S. Ann. Chem. 1952, 577, 139.

9. Rossiter, S. J. Chem. Soc. 1953, 3654.

10. Kamat, V. P.; Kirtany, J. K. Org. Prep. Proced. Int. 1994, $26,494$.

11. Wigfield, D. C.; Taymaz, K. Tetrahedron Lett. 1973, 4841. 
12. Berner, H.; Reinshagen, H. Monatsh. Chem. 1976, 107(1), 299.

13. Krayushkin, M. M.; Ivanov, S. N.; Lichitsky, B. V.; Dudinov, A. A. Russ. Chem. Bull., Int. Ed. 2001, 50, 2424.

14. Krayushkin, M. M.; Ivanov, S. N.; Lichitsky, B. V.; Dudinov, A. A. Russ. Chem. Bull. 2002, $51,1588$.

15. Ziegenbein, W.; Lang, W. Chem. Ber. 1960, 273.

16. Heinz, P. Chem. Ber. 1960, 2395.

17. Cagniant, M. P.; Cagniant, M.D. Bull. Soc. Chim. Fr. 1956, 1152. 